\title{
Effect of childhood trauma on adult depression and neuroendocrine function: sex-specific moderation by $\mathrm{CRH}$ receptor 1 gene
} \author{
Charles B. Nemeroff' ${ }^{1}$ Kerry J. Ressler ${ }^{1,3}$ and Elisabeth B. Binder ${ }^{1,4,5 *}$ \\ 1 Department of Psychiatry and Behavioral Sciences, Emory University School of Medicine, Atlanta, GA, USA \\ 2 VA Medical Center, Atlanta, GA, USA \\ 3 Yerkes National Primate Research Center, Atlanta, GA, USA \\ ${ }^{4}$ Department of Human Genetics, Emory University School of Medicine, Atlanta, GA, USA \\ ${ }_{5}^{5}$ Max Planck Institute of Psychiatry, Munich, Germany
}

Christine Heim ${ }^{1}$, Bekh Bradley ${ }^{1,2}$, Tanja C. Mletzko ${ }^{1}$,Todd C. Deveau', Dominique L. Musselman',

\section{Edited by:}

Larry J. Young, Emory University

School of Medicine, USA;

Yerkes National Primate Research

\section{Reviewed by:}

Carmine Pariante,

King's College London, UK

Richard Ebstein, The Hebrew

${ }^{*}$ Correspondence:

Elisabeth B. Binder,

Kraepelinstr. 2-10, 80804 Munich,

Germany.

e-mail: binder@mpipsykl.mpg.de; ebinder@emory.edu
Center, USA

University of Jerusalem, Israel

Max Planck Institute of Psychiatry

Variations of the corticotropin-releasing hormone receptor 1 (CRHR1) gene appear to moderate the development of depression after childhood trauma. Depression more frequently affects women than men. We examined sex differences in the effects of the CRHR1 gene on the relationship between childhood trauma and adult depression. We recruited 1,063 subjects from the waiting rooms of a public urban hospital. Childhood trauma exposure and symptoms of depression were assessed using dimensional rating scales. Subjects were genotyped for rs110402 within the CRHR1 gene. An independent sample of 78 subjects underwent clinical assessment, genotyping, and a dexamethasone/CRH test. The age range at recruitment was 18-77 years and 18-45, for the two studies respectively. In the hospital sample, the protective effect of the rs $110402 \mathrm{~A}$-allele against developing depression after childhood trauma was observed in men ( $N=424)$, but not in women ( $N=635)$. In the second sample, the rs 110402 A-allele was associated with decreased cortisol response in the dexamethasone/CRH test only in men. In A-allele carriers with childhood trauma exposure women exhibited increased cortisol response compared men; there were no sex differences in A-allele carriers without trauma exposure. This effect may, however, not be related to gender differences per se, but to differences in the type of experienced abuse between men and women. CRHR $\times$ environment interactions in the hospital sample were observed with exposure to physical, but not sexual or emotional abuse. Physical abuse was the most common type of abuse in men in this cohort, while sexual abuse was most commonly suffered by women. Our results suggest that the CRHR1 gene may only moderate the effects of specific types of childhood trauma on depression. Gender differences in environmental exposures could thus be reflected in sex-specific $C R H R 1 \times$ child abuse interactions.

Keywords: CRH receptor, gene x environment interaction, depression, dex-CRH test, polymorphism, HPA-axis, endocrine, genetics

\section{INTRODUCTION}

It is now well-established that adverse experience early in life, such as childhood abuse, neglect or loss, dramatically increases the risk of developing major depression in adulthood, particularly in the context of additional challenge (Bifulco et al., 1991; McCauley et al., 1997; Dube et al., 2001; Chapman et al., 2004). Considerable evidence from preclinical animal models and clinical studies suggests that stress early in life, during a period of heightened neural plasticity, leads to persistent hyperactivity of central nervous system (CNS) corticotropin-releasing hormone $(\mathrm{CRH})$ circuits and sensitization of hypothalamic-pituitary-adrenal (HPA) axis and autonomic nervous system responses to subsequent stress. (Ladd et al., 2000; Meaney and Szyf, 2005; Heim et al., 2008b; Lupien et al., 2009; McGowan et al., 2009). These persistent neurobiological consequences of early-life stress have been hypothesized to mediate the vulnerability to adult depression in victims of childhood trauma.
However, not all individuals who experience childhood trauma develop depression and some individuals remain resilient even when exposed to additional stressors in adolescence or adulthood. Identifying biological and psychological factors that contribute to resilience after childhood trauma may suggest novel targets for the prevention of depression. A considerable number of studies suggest that genetic factors moderate the relationship between earlylife stress and depression. Particular attention has been directed towards the role of a functional polymorphism in the promoter region of the serotonin transporter gene (5HTTLPR) in moderating the effects of childhood stress on adult depression (Caspi et al., 2003; Brown and Harris, 2008), though results are inconsistent across studies (Brown and Harris, 2008). A recent meta-analysis failed to corroborate the role of the 5HTTLPR in moderating the link between life stress and depression (Risch et al., 2009), though space constraints preclude a comprehensive discussion of 
this controversial area. Our group investigated gene-environment interactions in depression risk focusing on variations of genes involved in regulating stress response systems (Bradley et al., 2008). Recently, we reported that a haplotype of the $\mathrm{CRH}$ receptor 1 (CRHR1) gene, comprised of three single nucleotide polymorphisms (SNPs), exerts a protective effect against the development of depression after childhood trauma in two independent samples (Bradley et al., 2008). This finding has now been replicated in a second independent study from England, representing a trans-ethnic replication (Polanczyk et al., 2009). In addition, Tyrka et al. (2009) have reported that the early trauma $\times$ CRHR 1 genotype interaction is reflected in the cortisol response to combined dexamethasone/CRH stimulation. Thus, the CRHR1 gene may moderate the consequences of early adversity at the level of CRH-dependent neural transmission, though the functional relevance of the protective haplotype has not yet been ascertained.

In addition to the role of stress and genetic factors, there are important sex differences in the risk to develop depression. Major depression is twice as common in women as in men (Kessler et al., 1993; Kessler, 2003). Moreover, women more frequently develop depression in response to childhood trauma compared to men (Weiss et al., 1999). Sex differences in the risk for stress-related depression have been hypothesized to be, in part, due to direct effects of circulating estrogens on the CNS (Ter Horst et al., 2009), including hypothalamic CRH neurons (Vamvakopoulos and Chrousos, 1993). Taken together, it appears that a complex interaction between several intrinsic factors, i.e. genes and sex, and stressful experience underlie depression risk in most cases. Indeed, the CNS $\mathrm{CRH}$ system may be one substrate where the effects of these various dispositional and environmental factors are integrated.

Here we investigated interactions between the CRHR 1 gene and sex in moderating the effects of childhood trauma on adult depression in an expansion of the previously described sample recruited in the waiting rooms of a large public urban hospital (Bradley et al., 2008). In a second sample, recruited from the Atlanta metropolitan area, we ascertained effects of the CRHR1 gene and sex in moderating effects of early-life trauma at the level of an endophenotype of depression, i.e. neuroendocrine dysfunction. As a marker of neuroendocrine dysfunction, we measured cortisol response to a combined dexamethasone/CRH test, which is considered the most sensitive marker of HPA axis hyperactivity in depression (Ising et al., 2005). The dexamethasone/CRH test has previously been shown to be sensitive to detect familial (Holsboer et al., 1995) and environmental depression risk, including risk due to childhood trauma (Heim et al., 2008a). We hypothesized that a polymorphism of the CRHR1 gene moderates the effects of childhood trauma on adult depression and neuroendocrine dysfunction in a sex-specific manner.

\section{MATERIALS AND METHODS \\ STUDY 1 \\ Sample and sample recruitment}

Data from this study were collected as part of a larger study investigating the roles of genetic and environmental factors in predicting response to stressful life events in a predominantly African American, urban population of low socioeconomic status (Binder et al., 2008; Bradley et al., 2008). Research participants were approached while either waiting for their appointments in a general medical or obstetrical-gynecological clinic or while waiting with others who were scheduled for such appointments in a large public urban hospital. Subjects who indicated willingness to participate provided written informed consent, participated in a clinical interview, and provided a salivary sample for DNA extraction (described below). Participants were reimbursed $\$ 15.00$ for participation in this phase of the study. The presented data were obtained from the first 1392 African American subjects and include the 560 individuals described in an earlier report (Bradley et al., 2008). All procedures in this study were approved by the Institutional Review Boards of the Emory University School of Medicine and Grady Memorial Hospital. Demographic and clinical features of the sample, stratified by sex (62.2\% female), are presented in Table 1 .

\section{Clinical assessment and psychometric instruments}

Depressed mood was assessed with the 21-item self-report Beck Depression Inventory (BDI) (Beck et al., 1961). The BDI is a well validated, commonly used continuous measure of level of depressive symptoms. In this sample, the BDI had a standardized alpha coefficient $(\alpha)$ of $0.99(M=14.4, \mathrm{SD}=13.2)$ indicating high internal reliability. Symptoms of posttraumatic stress disorder (PTSD) were assessed using the modified PTSD Symptoms Scale (PSS)

Table 1 | Demographic and clinical features of men and women in study 1

\begin{tabular}{|c|c|c|c|}
\hline & $\begin{array}{l}\text { Female } \\
(N=639)\end{array}$ & $\begin{array}{l}\text { Male } \\
(N=424)\end{array}$ & $\begin{array}{l}\text { Level of } \\
\text { significance }\end{array}$ \\
\hline Age in years (M, SD) & $37.8(14.1)$ & $44.4(12.5)$ & $p<0.001$ \\
\hline Education level (\%) & & & $p=0.092$ \\
\hline$<12$ th grade & 26.0 & 24.9 & \\
\hline 12th grade & 38.2 & 37.4 & \\
\hline GED & 6.0 & 5.5 & \\
\hline $\begin{array}{l}\text { Some college or } \\
\text { technical school (Tech) }\end{array}$ & 20.8 & 19.0 & \\
\hline College/tech graduate & 8.0 & 11.2 & \\
\hline Graduate school & 0.9 & 2.1 & \\
\hline Relationship status (\%) & & & $p<0.001$ \\
\hline Never married & 64.2 & 53.0 & \\
\hline Married & 10.4 & 15.6 & \\
\hline Divorced & 11.1 & 20.8 & \\
\hline Separated & 6.9 & 8.0 & \\
\hline Widowed & 7.4 & 2.6 & \\
\hline Household income/ & & & \\
\hline month (\%) & & & $p=0.004$ \\
\hline$<500 \$$ & 38.7 & 39.6 & \\
\hline $500-1000 \$$ & 26.3 & 24.8 & \\
\hline $1000-2000 \$$ & 26.4 & 22.7 & \\
\hline$>2000 \$$ & 8.7 & 12.9 & \\
\hline Employment status (\%) & 34.3 & 30.2 & $p=0.16$ \\
\hline BDI total score (M, SD) & $13.4(11.6)$ & $11.3(10.7)$ & $p<0.001$ \\
\hline PSS total score (M, SD) & $11.1(11.7)$ & $10.5(11.4)$ & NS \\
\hline Moderate-severe abuse (\%) & & & $p=0.007$ \\
\hline Any & 43.7 & 35.5 & \\
\hline Physical & 22.2 & 21.0 & \\
\hline Sexual & 31.8 & 16.3 & \\
\hline Emotional & 20.0 & 17.0 & \\
\hline
\end{tabular}


(Falsetti et al., 1993). Consistent with prior literature, we summed the PSS frequency items ("0: not at all" to " $3: \geq$ Five times a week") to obtain a continuous measure of PTSD symptom severity ranging from $0-51$.

Childhood trauma was assessed using the 28 -item version of the Childhood Trauma Questionnaire (CTQ) (Bernstein et al., 2003). The CTQ is a self-report inventory assessing five types of childhood trauma, i.e. sexual, physical, and emotional abuse; and emotional and physical neglect. Studies have established the internal consistency, stability over time, and criterion validity of the 28 -item short form (Bernstein et al., 2003). The CTQ yields a total score and subscale scores for each type of childhood trauma. As prior research, we restricted analyses to the 3 abuse subscales (Bradley et al., 2008). Our data obtained from the abuse scales demonstrated high internal reliability ( $\alpha=0.99$ for physical abuse; $\alpha=0.94$ for sexual abuse; $\alpha=0.93$ for emotional abuse; $\alpha=0.98$ for the total of these three scales). Bernstein and Fink (1998) established cut-off scores for none, mild, moderate, and severe exposure levels for each type of childhood trauma. Based on the CTQ data, we dichotomized participants into two groups for each of the three categories of abuse (Physical, Sexual, and Emotional Abuse). For each category of abuse, subjects with scores in the none-mild range were classified as negative for exposure and subjects with scores in the moderatesevere range were classified as positive for exposure. Cut-off scores are $\geq 13$ for Emotional Abuse, $\geq 10$ for Physical Abuse, and $\geq 8$ for Sexual Abuse. We next created a composite variable across the three abuse types, dividing participants into (1) those with no exposure to any moderate-severe abuse and (2) those with moderate-severe exposure to at least one type of abuse.

\section{DNA extraction and genotyping}

Saliva was collected into Scope mouthwash $(N=46)$ or into Oragene saliva kits $(N=1346)$ (DNAGenotek, Inc.). DNA was extracted using the Qiagen M48 system and the Purelink 96 Genomic DNA Kit by Invitrogen (Cat \# K1821-04). SNP genotyping was performed as previously described (Binder et al., 2008; Bradley et al., 2008). Briefly, we examined the CRHR1 SNP rs110402, as this SNP had shown the strongest interaction with early trauma to predict depression in our previous publication. This SNP is also in high linkage disequilibrium with the other associated SNPs and can thus serve as tag-SNP (Bradley et al., 2008). The SNP was genotyped using a TaqMan allelic discrimination assay (Livak, 1999) developed for use on the 7900HT instrument (Applied Biosystems, Forster City, CA, USA), using predesigned and validated TaqMan assay reagent kits containing one pair of PCR primers and one pair of fluorescently labeled probes (Applied Biosystems; www.appliedbiosystems.com). PCR was performed in $5 \mu \mathrm{L}$ reaction volumes in 384-well plates and contained $5 \mathrm{ng}$ of DNA. The standard protocol provided with the kit was followed. Thermal cycler conditions were $95^{\circ} \mathrm{C}$ for $10 \mathrm{~min}$, 40 cycles of $95^{\circ} \mathrm{C}$ for $15 \mathrm{~s}$ and $60^{\circ} \mathrm{C}$ for $1 \mathrm{~min}$. The SDS 2.3 software was used for allelic discrimination. The call rate for the rs 110402 was $99.2 \%$ and 0 discordances were observed across duplicates.

\section{Data analysis}

Of the 1392 subjects, 1213 had completed both the BDI as well as the CTQ, and 1234 high quality DNA samples were obtained. This resulted in 1059 individuals with no missing data and thus data from 635 women and 424 men were analyzed using the statistical software SPSS version 15 . We used a general linear model to assess whether the genotype itself or in interaction with childhood abuse predicted current depressive symptoms. For CRHR1 variants, we investigated the effects of the most significant CRHR1 SNP, as identified in our previous study (Risch et al., 2009), rs110402, using an additive genetic model, with $\mathrm{A}$ being the protective allele. CRHR1 genotypes were coded as 0,1 and 2 (AA, AG and GG). Childhood abuse exposure was coded into a 2-level variable, as described above $(0=$ no type of moderate to severe child abuse; $1=$ at least one type of moderate to severe child abuse). Analyses were stratified by sex and adjusted for age, with rs110402 genotype, 2-level child abuse and their interaction term as predictors and the BDI total score as adults as outcome. In addition, we also investigated the same interaction in the whole sample with presence or absence of moderate to severe physical-, sexual- or emotional abuse alone. Differences between men and women were evaluated using contingency tables and general linear models. Analyses were 2-tailed with the level of significance set at 0.05 .

\section{STUDY 2}

\section{Subjects}

The sample of the neuroendocrine study was comprised of 78 subjects ( 25 men and 53 women), aged $18-45$ years $(M=29.7$, $\mathrm{SD}=7.5$ years). Subjects were recruited as part of the larger Conte Center for the Psychobiology of Early-Life Trauma (MH58922) and, by definition, included subjects with/without exposure to childhood trauma before the age of 13 years and with/without a diagnosis of major depression. For this study, subjects were classified into those with versus those without childhood trauma exposure, as described below. Exclusion criteria were current medical illness, lifetime psychosis or bipolar disorder, alcohol or substance abuse within 6 months or eating disorders within the past year. None of the participants was in current psychiatric treatment or currently received medications. Heavy smokers ( $>20$ cigarettes/ day) were excluded. All subjects were recruited from responses to advertising in local newspapers and the transportation system and screened for eligibility. Eligible subjects were invited and paid for participation. After description of the study to participants, written informed consent was obtained. The study was approved by the Institutional Review Board of Emory University School of Medicine.

\section{Methods}

Subjects were admitted as inpatients to the Atlanta Clinical and Translational Science Institute, Emory University Hospital Clinical Interaction Site, which provides a standardized setting for clinical studies. Exposure to childhood trauma was assessed using the CTQ (Bernstein et al., 2003). Similar to Study 1, we used moderate-severe cut-off scores on each subscale to classify cases as positive or negative for a history of significant trauma on each of the five subscales ( $\geq 13$ for Emotional Abuse; $\geq 10$ for Physical Abuse; $\geq 8$ for Sexual Abuse; $\geq 15$ for Emotional Neglect; and $\geq 10$ for Physical Neglect). (Bernstein and Fink, 1998). Cases classified as positive on any one subscale were assigned to the group of cases with exposure to childhood trauma, whereas cases classified as negative on all subscales were assigned to the group of cases without exposure to significant 
childhood trauma. A total of 51 subjects were classified as positive for a history of childhood trauma and 27 were classified as negative for a history of childhood trauma.

On the first day after hospital admission, subjects provided a blood sample for genotyping. Samples were assayed for the rs 110402 SNP of the CRHR1 gene, as described above. In this cohort, 28 subjects carried the GG, 34 the AG, and 16 the AA genotype. Subjects were further categorized based on the presence of A alleles of the rs110402, resulting in 50 A-allele carriers (AA or AG genotype) and 28 non-carriers (GG genotype).

The dexamethasone/CRH test was performed on the second inpatient day. In this test, a dose of $1.5 \mathrm{mg}$ dexamethasone (Roxane Laboratories, Columbus, $\mathrm{OH}$ ) was administered orally at 11 p.m. On the following day, antecubital venous catheters were inserted at 12 p.m. and kept patent by saline infusion. Post-dexamethasone blood samples were collected at 2 p.m. (-60 min), 2:30 p.m. (-30 $\mathrm{min})$, and 3 p.m. (0 min). Immediately after the 3 p.m. sample, a bolus intravenous injection of $1 \mu \mathrm{g} / \mathrm{kg}$ ovine CRH (Ferring, Suffern, NY, USA) was administered. Blood samples were collected at 5, 15, 30, 60, 90, and $120 \mathrm{~min}$ after $\mathrm{CRH}$ injection. Blood was collected in EDTA tubes, placed immediately on ice, and centrifuged at $4^{\circ} \mathrm{C}$ for $10 \mathrm{~min}$ at 3,000 $\mathrm{rpm}$. Plasma was separated, coded and stored at $-80^{\circ} \mathrm{C}$. Plasma cortisol concentrations were measured using a commercial radioimmunoassay (DiaSorin, Stillwater, MN, USA). Assay sensitivity was $0.04 \mu \mathrm{g} / \mathrm{dl}$. Intra-assay variability was $6.7 \%$ and interassay variability was $7.1 \%$.

\section{Data analysis}

As noted above, subjects were grouped according to the following factors: History of childhood trauma (present vs. absent), sex (male vs. female), and CRHR1 rs110402 A-allele carrier status (GG vs. $\mathrm{AG}+\mathrm{AA}$ ). The protective/A allele carriers (genotypes AA and AG) were merged into one group to increase power, which was necessary due to the small sample size. Cortisol data were compared between various groups using repeated measures analysis of variance. The sample size did not allow for simultaneous testing of the 4-way or various 3-way interaction effects between childhood trauma, sex, genotype, and time. We therefore computed hypothesis-driven 2-way interaction models to test for effects of interest: We first tested the effects of rs110402 on cortisol response in the dexamethasone/ $\mathrm{CRH}$ test for women and men, separately, to test the hypothesis that the protective effect of the A allele occurs only in men. We then selected A allele carriers with and without exposure to childhood trauma and tested for sex by time interaction effects on cortisol response in order to test the hypothesis that the protective effect of the A allele occurs only in men exposed to childhood trauma, whereas this effect is not observed in non-exposed men or women exposed to childhood trauma. Significant effects were confirmed when controlling for current or lifetime major depression. Analyses were 2-tailed with the level of significance set at 0.05 .

\section{RESULTS}

\section{STUDY 1}

\section{Sex differences in demographic and clinical variables}

Demographic and clinical features of men and women in the sample are presented in Table 1. Significant differences between men and women were observed for marital status and education, but not household income or employment status. The mean BDI total score was significantly higher in women than in men, and women had experienced significantly more childhood abuse than men. A proportion of $43.7 \%$ of women versus $35.5 \%$ of men had experienced any type of moderate-severe type of childhood abuse. This difference was carried by the higher prevalence of moderate-severe sexual abuse in women. Severity of PTSD symptoms did not differ between men and women. The severity of physical, sexual or emotional abuse was not significantly different between men and women (data not shown). However, of the women with at least one type of moderate to severe abuse, $73.0 \%$ had experienced sexual abuse, while this was only true for $43.0 \%$ of male participants. Physical abuse was the most common type of abuse in men (Table 1). In men who had been exposed to physical abuse, $30.4 \%$ had also experienced sexual abuse, while this proportion was much higher in females, with $62.8 \%$.

\section{Interaction between rs110402 and child abuse stratified by sex}

We tested the interaction of rs1 10402 genotype and any childhood abuse on current depressive symptoms in female and male subjects, separately. Analyses were conducted in 424 male and 635 female subjects (Figure 1). In women (Figure 1A), childhood abuse exposure was a significant predictor $\left(F_{1,634}=25.0, p<0.0001\right)$, but there was neither a main effect of genotype nor an interaction effect of genotype by sex. In men (Figure 1B), however, there was a significant main effect of childhood abuse exposure $\left(F_{1,423}=8.20\right.$, $p=0.004)$ as well as a significant interaction effect of childhood abuse exposure by rs110402 genotype $\left(F_{2,423}=3.50, p=0.031\right)$, whereas there was no main effect of the genotype. Specifically, men homozygous for the A allele were most protected from developing depression after childhood abuse.

\section{Interaction between rs110402 and different types of child abuse and role of sex}

In the combined gender sample, only the interaction of physical abuse with rs110402 but not with the presence of emotional or sexual abuse was statistically significant (Figure 2). We observed significant effects of exposure to all three types of abuse on depressive symptoms (physical abuse $F_{1,1058}=17.5, p<0.001$, sexual abuse $F_{1,1058}=11.7, p=0.001$, emotional abuse $F_{1,1058}=72.7, p<0.001$ ). A significant main effect of rs1 10402 on depressive symptoms was seen in all three analyses $(p<0.05)$. The significant interaction of the SNP with exposure to childhood abuse remained only with the presence of physical abuse $\left(F_{2,1058}=5.45, p=0.004\right)$. To exclude that these results were due to different distribution of types of abuse between the sexes, we reran analyses in the male subsample and, again, only the interaction with physical abuse $\left(F_{2,423}=4.1\right.$, $p=0.018)$, but not the other two abuse types $\left(F_{2,423}=0.09, p=0.91\right.$ for sexual abuse and $F_{2,423}=1.43, p=0.24$ for emotional abuse) was statistically significant. In women, none of the interactions reached statistical significance.

\section{STUDY 2}

\section{Sex difference in the effect of rs110402 on cortisol response}

We first tested the hypothesis that there are sex differences in the effects of the protective CRHR1 rs110402 A allele on cortisol response to the dexamethasone/CRH test. For statistical 
A

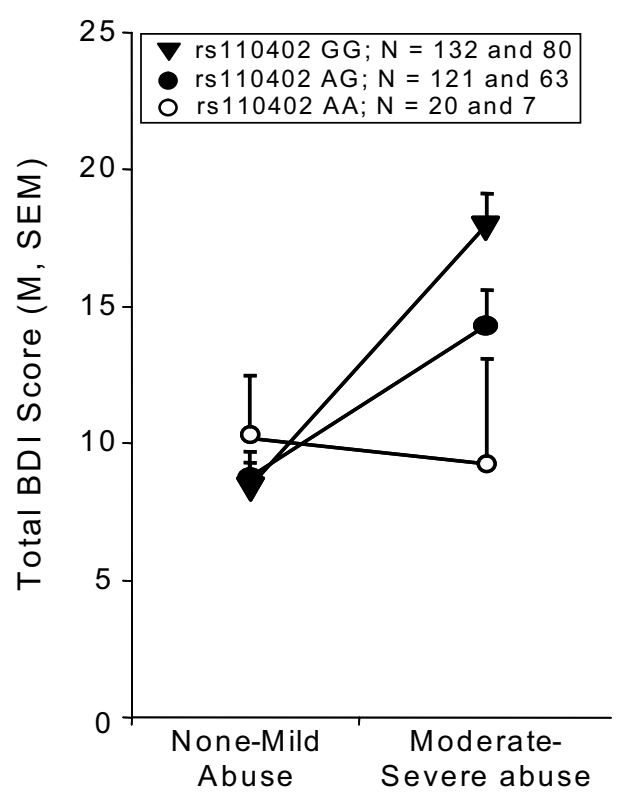

FIGURE 1 | Interaction of CRHR1 rs110402 genotype and childhood abuse exposure on current depressive symptoms in men $(N=424)$ and women ( $N=\mathbf{6 3 5})$. Graph depicts mean (SEM) total BDI scores in subjects grouped according to rs110402 allele status (GG, AG, AA) and exposure to none-mild versus moderate-severe childhood abuse. (A) In men, there was a significant main effect of childhood abuse exposure $\left(F_{1,423}=8.20, p=0.004\right)$ and $a$ significant interaction effect of rs 110402 by childhood abuse exposure

\section{B $\quad$ Female Subjects}

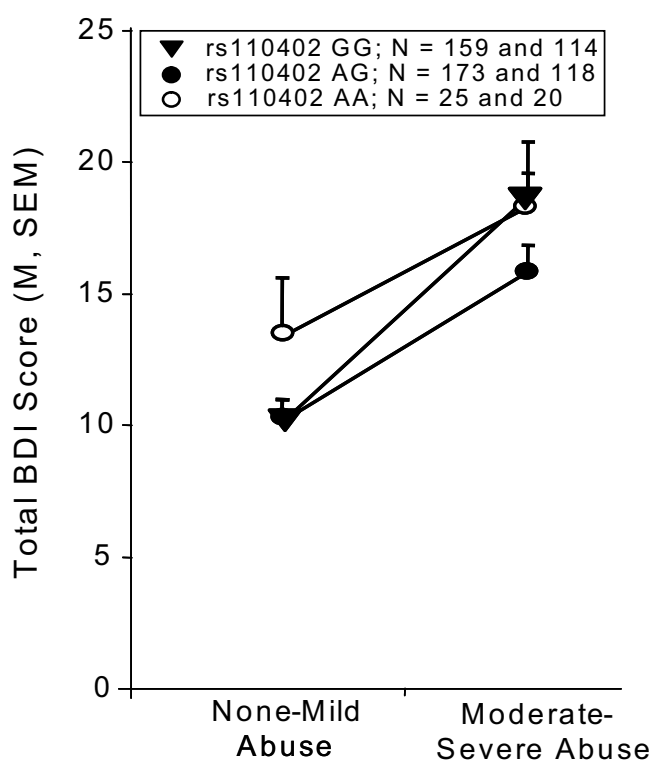

$\left(F_{2,423}=3.50, p=0.031\right)$ on BDI scores. (B) In women, there was a significant main effect of childhood abuse exposure on BDI score $\left(F_{1,634}=25.0\right.$,

$p<0.0001$ ), but no significant gene-environment interaction of rs110402 and childhood abuse exposure on BDI scores. The genotype distribution is given in the figure legend, with the first number representing the $\mathrm{N}$ in the non-mild abuse group and the second number the $N$ in the moderate to severe abuse exposure group. analyses, we pooled subjects carrying 1 or $2 \mathrm{~A}$ alleles ( $\mathrm{GG}+\mathrm{AA}$ ) and compared A allele carriers with GG carriers. For informative reasons, rs110402 subgroups (AA, AG, and GG) are presented separately in Figure 3. For women (Figure 3A), repeated measures ANOVA revealed a significant main effect of the time factor, indicating similar increases in cortisol concentrations after CRH injection in all groups $\left(F_{10,500}=68.486, p<0.001\right)$. However, there was neither a significant main effect of genotype, nor a genotype by time interaction effect, on plasma cortisol concentrations in women. For men (Figure 3B), repeated measures ANOVA revealed a significant interaction effect of genotype by time $\left(F_{10,230}=2.225\right.$, $p=0.017)$ : Men carrying one or two rs $110402 \mathrm{~A}$ allele demonstrated markedly decreased plasma cortisol responses in the dexamethasone/CRH test compared to men who were homozygous rs110402 GG-allele carriers. All effects remained significant when controlling for current or lifetime major depression.

\section{Sex differences in the effect of childhood trauma on cortisol response in rs110402 A allele carriers}

We next selected carriers of the rs110402 A allele (AG or AA) to test the hypothesis that the effect of the A allele in decreasing cortisol response in the dexamethasone/CRH test exclusively occurs in men with histories of childhood trauma, but not in men without such histories or women with or without childhood trauma history (Figure 4). For A allele carriers with childhood trauma exposure (Figure 4A), repeated measures ANOVA revealed a significant interaction effect of sex by time $\left(F_{10,300}=3.652, p<0.001\right)$, indicating that female A allele carriers exposed to childhood trauma demonstrated increased cortisol response in the dexamethasone/CRH test compared to male A allele carriers exposed to childhood trauma who demonstrated markedly low response. Among A allele carriers without childhood trauma exposure (Figure 4B), there only was a significant main effect of the time factor $\left(F_{10,160}=15.173, p<0.001\right)$, indicating similar increases in plasma cortisol concentrations in all groups. However, there were no sex differences in neuroendocrine response among A allele carriers in the absence of childhood trauma. All effects remained significant when controlling for current or lifetime major depression.

\section{DISCUSSION}

We evaluated interactions between the CRHR1 gene and sex in moderating the well-known association between childhood trauma and adult depression (Bifulco et al., 1991; McCauley et al., 1997; Ladd et al., 2000; Dube et al., 2001; Chapman et al., 2004). Previous research has suggested that allelic variation of the CRHR1 gene, particularly the rs1 10402 SNP located in intron 2 of the gene, protects against developing adult depression in the presence of moderatesevere childhood abuse (Bradley et al., 2008; Polanczyk et al., 2009). Another line of research has provided longstanding evidence for a marked sex difference in the risk to develop depression, inasmuch as women more frequently suffer from depression in general (Kessler et al., 1993; Kessler, 2003) and more frequently develop depression in 


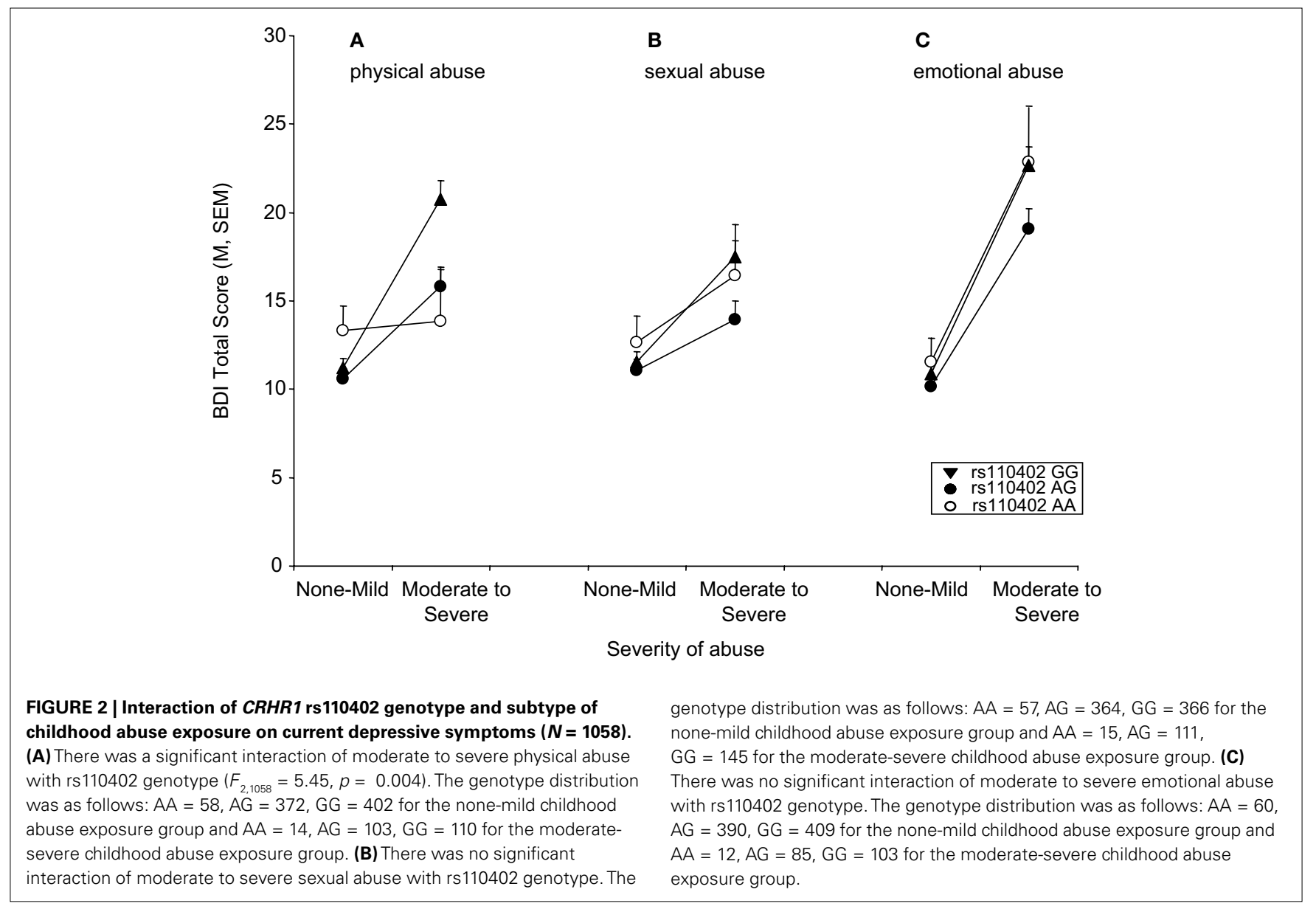

A

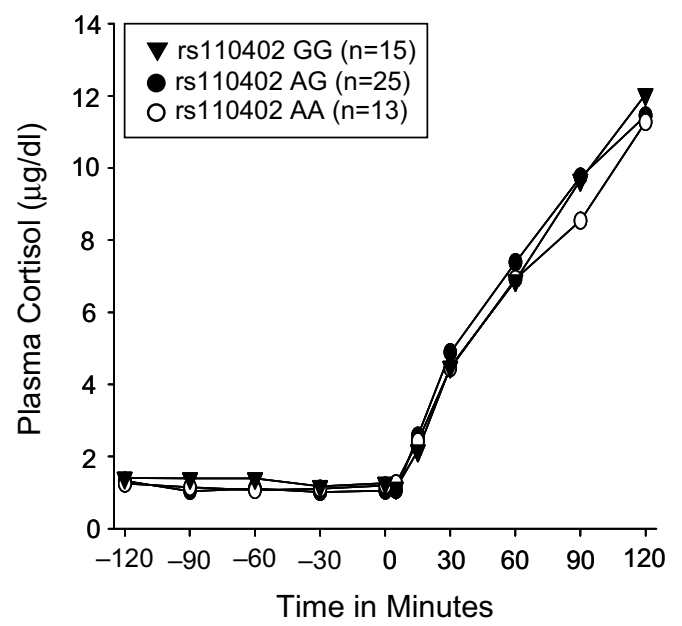

FIGURE 3 | Effect of CRHR1 rs110402 on cortisol response in the dexamethasone/CRH test in women $(\boldsymbol{N}=53)$ versus men $(\boldsymbol{N}=\mathbf{2 5})$. Graph depicts mean plasma cortisol concentrations $(\mu \mathrm{g} / \mathrm{dl})$ in subjects grouped according to rs 110402 allele status (GG, AG, AA). Subjects with 1 or 2 A alleles ( $A G$ or $A A$ ) were pooled for statistical analysis and compared with subjects carrying GG-alleles (see text). (A) In women, repeated measures
B Men

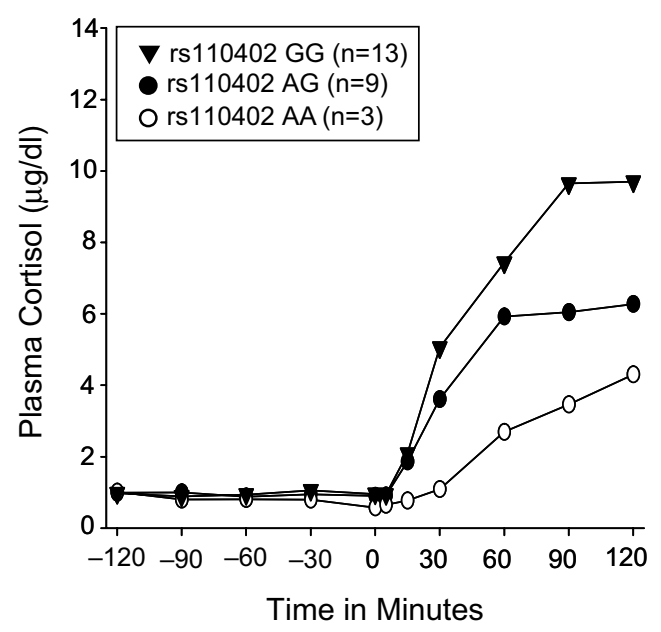

ANOVA only revealed a significant main effect for the time factor $\left(F_{10,500}=68.486, p<0.001\right)$, indicating similar increases in cortisol concentrations in all groups. (B) In men, repeated measures ANOVA revealed a significant interaction of time by genotype $\left(F_{10,230}=2.225, p=0.017\right)$, indicating decreased response in men carrying the $A$ allele compared to men homozygous GG. 
A

With ELS

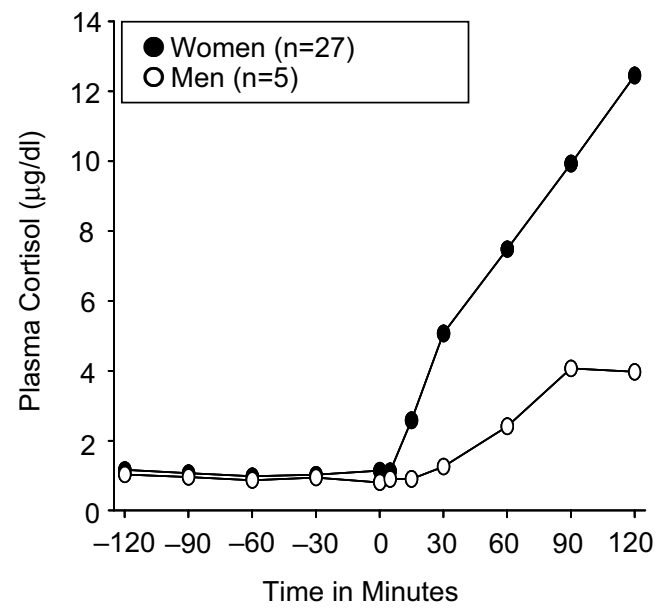

FIGURE 4 | Effect of sex on cortisol response in dexamethasone/CRH test in carriers of the CRHR1 rs $110402 \mathrm{~A}$ allele with $(N=32)$ and without $(N=18)$ histories of childhood trauma. Graph depicts mean plasma cortisol

concentrations $(\mu \mathrm{g} / \mathrm{dl})$ in subjects grouped by sex. (A) In A allele carriers with

moderate-severe childhood trauma exposure, repeated measures ANOVA revealed
B

Without ELS

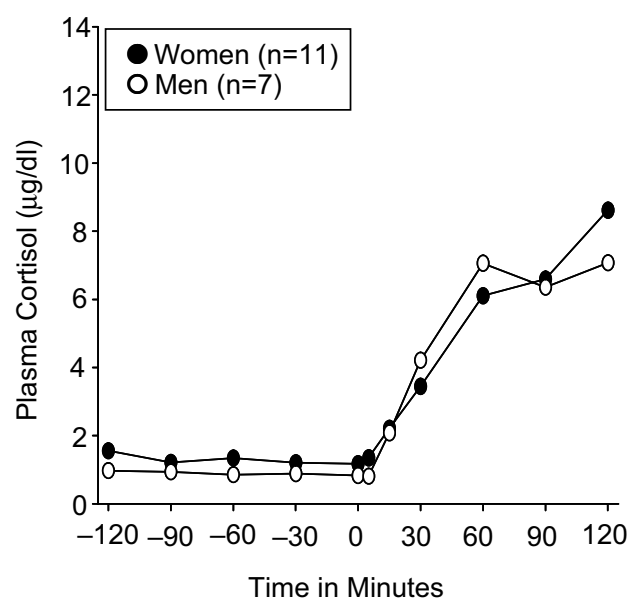

a significant interaction effect of time by genotype $\left(F_{10,300}=3.652, p<0.001\right)$, indicating increased response in women compared to men. (B) In A allele carriers without moderate-severe childhood trauma exposure, repeated measures ANOVA only revealed a significant main effect of the time factor $\left(F_{10,160}=15.173, p<0.001\right)$, indicating similar increases in cortisol concentrations in women and men. relation to childhood trauma (Weiss et al., 1999)compared to men. We therefore tested the hypothesis that the protective effect of the CRHR1 gene against developing depression after childhood trauma is specific to one of these sexes, and that one mechanism that mediates this effect involves a decrease $\mathrm{CRH}$-dependent neurotransmission.

Our results are consistent with this hypothesis: We demonstrate in a large urban public hospital sample that the A allele of the rs110402 SNP is associated with decreased symptoms of depression among male subjects exposed to moderate-severe childhood abuse exposure, whereas this protective effects is not observed in female subjects with childhood abuse exposure. In a second sample of subjects recruited from the Atlanta metropolitan area, we confirm this sex by gene interaction at the level of an endophenotype of depression, i.e. cortisol response in the dexamethasone/CRH test, which is considered a sensitive marker of CRH/HPA axis hyperactivity in depression (Vamvakopoulos and Chrousos, 1993; Ter Horst et al., 2009). Specifically, although allelic variation in the rs110402 SNP did not affect cortisol response to the dexamethasone/CRH test in women, male rs110402 A allele carriers had markedly decreased cortisol responses compared to male GG genotype carriers. A second analysis within the sample of A allele carriers, revealed that women with childhood trauma exposure exhibited increased cortisol response compared to men with childhood trauma exposure. There were no differences between male and female A allele carriers in the absence of childhood trauma. These results indeed suggested that the protective effect of rs110402 occurs specifically in men and becomes only effective in the presence of childhood trauma, resulting in decreased $\mathrm{CRH}$-dependent neuroendocrine reactivity and, hence, decreased symptoms of depression.

Of note, the number of rs110402 A alleles appears to demonstrate an inverse dose-response relationship with adult depressive symptoms in the hospital sample and the level of cortisol response to the dexamethasone/CRH test in the second sample. Thus, the observed genetic effects were more pronounced among subjects who were homozygous AA allele carriers than among subjects who were AG genotype carriers. It should be noted that we observed these concurring dose-response effects of rs110402 on depression and neuroendocrine function across two completely independent samples of subjects. These effects were present in spite of differences between the two studies: First, the urban hospital sample included in this report was comprised of African-Americans, whereas the second sample undergoing neuroendocrine testing was racially diverse. Second, the definition of the exposure variable was different between the two studies. The urban hospital sample categorized subjects based on the presence or absence of moderate-severe sexual, physical or emotional abuse, whereas the sample with neuroendocrine measures additionally considered the presence or absence of emotional or physical neglect. Therefore, the non-exposed subjects in the second sample did not have any significant childhood trauma, whereas the non-abused subjects in the hospital sample might have had experienced neglect to some extent. However, it was not possible in the latter sample to stratify based on CTQ neglect scales because these scores were not valid in this socioeconomically disadvantaged sample (see Bradley et al., 2008 for discussion).

In previous CRHR1 gene $\times$ environment studies (Bradley et al., 2008; Polanczyk et al., 2009), a haplotype of allelic variations (TAT) across 3 SNPs within the CRHR1 gene, including rs242924, rs110402, and rs7209436, was particularly protective against developing depression. All three SNPs are in very high linkage disequilibrium in both African American as well as European American samples, so that there is an almost complete overlap of rs110402 A allele carriers and TAT carriers. Interactions in the males of the expanded hospital sample or with physical abuse in the 
whole sample are thus in the same direction as the ones previously reported (Bradley et al., 2008). Due to the high degree of linkage disequilibrium, it is difficult to pinpoint one of these SNPs as the functional variant. The region tagged by these variants spans a region from the $5^{\prime}$ putative promoter area to exon 3 . Additional fine-mapping and re-sequencing will thus be necessary to identify the true functional variant.

While there are a number of preclinical studies that would support male-specific effects for this interaction (Sánchez et al., 2005; Mueller and Bale, 2008; Weiser et al., 2008), the recently published study by Polanczyk et al. (2009) is at odds with the notion that the protective effect of CRHR1 rs110402 is specific to men. Polanczyk et al. replicate the early trauma $\times$ CRHR 1 SNP interactions on adult depression in the $100 \%$ female E-Risk study from Great Britain, but not in the mixed-sex Dunedin study from New Zealand. In addition, in the latter study, no sex-specific interactions were observed. In order to reconcile these results with our observations, we further investigated potential interactions between CRHR1 SNP and abuse types. Men and women in Study 1 demonstrated differences in the frequency of the types of experienced abuse. As shown in other cohorts (Gilbert et al., 2009), sexual abuse was much more frequent in women than in men in Study 1 , whereas the most frequent type of abuse in men was physical abuse, mostly in the absence of sexual abuse (Table 1). On the other hand, most women who had experienced physical abuse had also experienced sexual abuse. We thus re-analyzed the $C R H R 1 \times$ abuse interaction with the specific subtypes of abuse and found that only physical abuse, but not emotional or sexual abuse, showed a significant interaction with CRHR1 genotype on current depressive symptoms. This could suggest that the observed male-specific effects in our cohort are driven by the fact that physical abuse is more prevalent in this group than in women. In addition, prior analyses of data (unpublished) from subjects in study 1 indicate that across both males and females, physical abuse has an earlier average self-reported age at time of first experience than does sexual abuse and these analyses also indicate that across both males and female participants the self-reported frequency of physical abuse was higher than that of sexual abuse. Thus, given the high rates of physical abuse in male participants in study 1 it is possible that our finding of male-specific gene $x$ environment interactions may be associated with an earlier onset and more frequent childhood stressor. These trauma- or trauma timing-specific interactions may in part be the underlying cause for the sex-specific neuroendocrine effects. Because of a small sample size and multiple exposures to different types of trauma, we could not verify this hypothesis in the second sample. Of note, the observation that the interaction of the CRHR1 polymorphism is most pronounced with physical abuse is in line with a previous study showing that long-term changes in cerebrospinal $\mathrm{CRH}$ are only seen with physical but not sexual abuse (Heim et al., 2008b).

A limitation of our study is that the size of the sample with neuroendocrine measures was too small to model 3- or 4-way interaction effects of CRHR1 gene, sex, childhood abuse, and time on cortisol concentrations. We also could not selectively assess the impact of the different types in that sample. Our results thus need to be verified in larger samples modeling full interaction effects in future studies. The putative interaction between rs1 10402 genotype and childhood abuse in males were, however, in the same direction as the ones reported by Tyrka et al. (2009). In both analyses, carriers of the protective A-allele exposed to childhood trauma displayed less HPA axis hyperactivity than individuals homozygous for the G-allele. Although we controlled for the impact of current or lifetime major depression, future studies in larger samples should include major depression and other Axis I disorders in a multifactorial model. However, the fact that similar findings have been reported in individuals without any current psychiatric disorder (Tyrka et al., 2009) suggests that the reported interaction maybe independent of current psychiatric symptoms. In addition, the comparability of our endocrine findings to the ones reported by Tyrka et al. (2009) further supports the trans-ethnic validity of this gene-environment interaction as our sample was of mixed ethnicity compared to the white non-Hispanic sample previously reported (Tyrka et al., 2009).

A second limitation is our reliance on retrospective and uncorroborated self-reports of childhood experiences. Problems concerning the credibility of self-reports of childhood trauma include simple forgetting, non-awareness, non-disclosure, and reporting biases due to mood states (Hardt and Rutter, 2004). Moreover, declarative biographical memory before the age of 5 years is sparse perhaps because the hippocampus is not fully developed. Early trauma itself leads to hippocampal damage, which might impair recall of childhood experiences (Bremner, 1999). A recent metaanalysis of studies using external corroboration of self-reports revealed that false negatives are more frequent than false positives, leading to downward biases in estimated associations between early adversity and outcome variables; the use of validated psychometric instruments and focus on moderate-severe early trauma increased validity of self-reports (Hardt and Rutter, 2004). Of note, the $C R H R 1$ gene $\times$ abuse interaction might also be dependent on the emotional connotation of the trauma experience that is evoked by the questions of the CTQ, but not other types of early trauma questionnaires that employ more factual questions (Polanczyk et al., 2009).

In conclusion, our results lend preliminary support of the hypothesis that the CRHR1 gene and sex interact in moderating effects of childhood trauma on adult depression. This gene-sexenvironment interaction was observed on the symptoms as well as endocrine level. Accordingly, we observed decreased cortisol reactivity in male rs110402 A allele carriers with childhood trauma exposure. Further analyses revealed that these sex-specific interactions may be carried by the type of experienced abuse. A significant interaction was only observed with physical abuse and this abuse is the most common type of abuse in men in our samples and is experienced more frequently and at an earlier age than sexual abuse. In addition, physical, but not sexual abuse has been associated with long-term increases of CSF CRH concentrations (Heim et al., 2008b). These findings underline the importance of an indepth characterization of childhood abuse and other environmental stressors, including age of onset, frequency and severity in gene $\times$ environment predictors of psychopathology.

Overall, longitudinal studies are needed to provide information on the causal relationship between childhood trauma and adult depression, as well as the moderators and mediators of this relationship. Such studies may not only have the potential to 
reconcile inconsistent neurobiological findings in depression, but will ultimately help elucidate the pathophysiology, identify subtypes, and devise strategies for the prevention and treatment of depression. Our findings further emphasize the need to integrate complex interactions of the various dispositional and environmental contributors to depression.

\section{REFERENCES}

Beck, A. T., Ward, C. H., Mendelson, M., Mock, J., and Erbaugh, J. (1961). An inventory for measuring depression. Arch. Gen. Psychiatry 4, 561-571.

Bernstein,D.,andFink,L. (1998).Childhood Trauma Questionnaire.A Retrospective Self-Report Questionnaire and Manual. San Antonio, The Psychological Corporation.

Bernstein,D.P.,Stein,J.A.,Newcomb, M. D., Walker, E., Pogge, D., Ahluvalia, T., Stokes, J., Handelsman, L., Medrano, M., Desmond, D., and Zule, W. (2003). Development and validation of a brief screening version of the Childhood Trauma Questionnaire. Child Abuse Negl. 27, 169-190.

Bifulco, A., Brown, G. W., and Adler, Z. (1991). Early sexual abuse and clinical depression in adult life. Br. J. Psychiatry 159, 115-122.

Binder, E. B., Bradley, R. G., Liu, W., Epstein, M.P.,Deveau,T.C.,Mercer, K. B., Tang, Y., Gillespie, C. F., Heim, C. M., Nemeroff, C. B., Schwartz,A. C., Cubells, J. F., and Ressler, K. J. (2008). Association of FKBP5 polymorphisms and childhood abuse with risk of posttraumatic stress disorder symptoms in adults. J. Am. Med. Assoc. 299, 1-15.

Bradley, R. G., Binder, E. B., Epstein, M. P., Tang, Y., Nair, H. P., Liu, W., Gillespie, C. F., Berg, T., Evces, M., Newport,D.J.,Stowe, Z. N.,Heim, C. M., Nemeroff, C. B., Schwartz,A., Cubells, J. F., and Ressler, K. J. (2008). Influence of child abuse on adult depression: moderation by the corticotropinreleasing hormone receptor gene. Arch. Gen. Psychiatry 65, 190-200.

Bremner, J. D. (1999). Does stress damage the brain? Biol. Psychiatry 45, 797-805.

Brown, G. W., and Harris, T. O. (2008). Depression and the serotonin transporter 5-HTTLPR polymorphism: a review and a hypothesis concerning gene-environment interaction. J. Affect. Disord. 111, 1-12.

Caspi, A., Sugden, K., Moffitt, T. E., Taylor, A., Craig, I. W., Harrington, H., McClay, J., Mill, J., Martin, J., Braithwaite,A., and Poulton, R. (2003). Influence of life stress on depression: moderation by a polymorphism in the 5-HTT gene. Science 301, 386-389.

Chapman, D. P., Whitfield, C. L., Felitti, V. J., Dube, S. R., Edwards, V. J., and Anda, R. F. (2004). Adverse childhood experiences and the risk of depressive disorders in adulthood. J. Affect. Disord. 82, 217-225.

Dube, S. R., Anda, R. F., Felitti, V. J., Chapman, D. P., Williamson, D. F., and Giles, W.H. (2001). Childhood abuse, household dysfunction, and the risk of attempted suicide throughout the life span: findings from the Adverse Childhood Experiences Study. JAMA 286, 3089-3096.

Falsetti, S. A., Resnick, H. S., Resick, P. A., and Kilpatrick, D. (1993). The Modified PTSD Symptom Scale: a brief selfreport measure of posttraumatic stress disorder. Behav. Therapist 16, 161-162.

Gilbert, R., Widom, C. S., Browne, K., Fergusson, D., Webb, E., and Janson, S. (2009). Burden and consequences of child maltreatment in high-income countries. Lancet 373, 68-81.

Hardt, J., and Rutter, M. (2004).Validity of adult retrospective reports of adverse childhood experiences: review of the evidence. J. Child Psychol. Psychiatry 45, 260-273.

Heim, C., Mletzko, T., Purselle, D., Musselman, D. L., and Nemeroff, C. B. (2008a). The dexamethasone/corticotropin-releasing factor test in men with major depression: role of childhood trauma. Biol. Psychiatry 63, 398-405.

Heim, C., Newport, D. J., Mletzko, T., Miller, A. H., and Nemeroff, C. B. (2008b). The link between childhood trauma and depression: insights from HPA axis studies in humans. Psychoneuroendocrinology 33, 693-710.

Holsboer, F., Lauer, C. J., Schreiber, W., and Krieg, J. C. (1995). Altered hypothalamic-pituitary-adrenocortical regulation in healthy subjects at high familial risk for affective disorders. Neuroendocrinology 62, 340-347.

Ising, M., Künzel, H. E., Binder, E. B., Nickel, T., Modell, S., and Holsboer F. (2005). The combined dexamethasone/CRH test as a potential surrogate marker in depression. Prog. Neuropsychopharmacol. Biol. Psychiatry 29, 1085-1093.

Kessler, R. C. (2003). Epidemiology of women and depression. J. Affect. Disord. 74, 5-13.

Kessler,R.C.,McGonagle, K.A.,Swartz, M., Blazer, D. G., and Nelson, C. B. (1993). Sex and depression in the National Comorbidity Survey. I: lifetime prevalence, chronicity and recurrence. J. Affect. Disord. 29, 85-96.

\section{CONTRIBUTIONS}

This research was funded by MH071537 (Kerry J. Ressler), MH58922 -project 6 (Charles B. Nemeroff/Christine Heim), and a NARSAD Young Investigator Award (Elisabeth B. Binder), and American Suicide Foundation Young Investigator Grant (Bekh Bradley) and an Emory University Research Committee Grant (Bekh Bradley).

Ladd,C.O.,Huot, R.L., Thrivikraman, K. V., Nemeroff, C. B., Meaney, M. J., and Plotsky, P. M. (2000). Long-term behavioral and neuroendocrine adaptations to adverse early experience. Prog. Brain Res. 122, 81-103.

Livak, K. J. (1999). Allelic discrimination using fluorogenic probes and the 5 ' nuclease assay. Genet. Anal. 14 143-149.

Lupien, S. J., McEwen, B. S., Gunnar, M. R. and Heim, C. (2009). Effects of stress throughout the lifespan on the brain, behaviour and cognition. Nat. Rev. Neurosci. 10, 434-445.

McCauley, J., Kern, D. E., Kolodner, K., Dill, L.,Schroeder,A.F.,DeChant, H. K. Ryden, J., Derogatis, L. R., and Bass, E. B. (1997). Clinical characteristics of women with a history of childhood abuse: unhealed wounds. JAMA 277, 1362-1368.

McGowan,P.O.,Sasaki,A., D’Alessio, A. C. Dymov, S., Labonté, B., Szyf, M. Turecki, G., and Meaney, M. J. (2009). Epigenetic regulation of the glucocorticoid receptor in human brain associates with childhood abuse. Nat. Neurosci. 12, 342-348.

Meaney, M. J., and Szyf, M. (2005) Environmental programming of stress responses through DNA methylation: life at the interface between a dynamic environment and a fixed genome. Dialogues Clin. Neurosci. 7, 103-123.

Mueller, B. R., and Bale, T. L. (2008) Sex-specific programming of offspring emotionality after stress early in pregnancy. J. Neurosci. 28 9055-9065.

Polanczyk, G., Caspi, A., Williams, B., Price, T. S., Danese, A., Sugden, K. Uher, R., Poulton, R., and Moffitt, T. E. (2009). Protective effect of CRHR1 gene variants on the development of adult depression following childhood maltreatment: replication and extension. Arch. Gen. Psychiatry 66, 978-985.

Risch, N., Herrell, R., Lehner, T., Liang, K. Y., Eaves, L., Hoh, J., Griem, A., Kovacs, M., Ott, J., and Merikangas, K. R. (2009). Interaction between the serotonin transporter gene (5-HTTLPR), stressful life events, and risk of depression: a meta-analysis. J. Am. Med. Assoc. 301, 2462-2471.

Sánchez, M. M., Noble, P. M., Lyon, C. K., Plotsky,P.M.,Davis, M.,Nemeroff, C. B., and Winslow, J. T. (2005). Alterations in diurnal cortisol rhythm and acoustic startle response in nonhuman primates with adverse rearing. Biol. Psychiatry 57, 373-381.

Ter Horst, G. J., Wichmann, R., Gerrits, M., Westenbroek, C., and Lin, Y. (2009). Sex differences in stress responses: focus on ovarian hormones. Physiol. Behav. 97, 239-249.

Tyrka, A. R., Price, L. H., Gelernter, J., Schepker, C., Anderson, G. M., and Carpenter, L. L. (2009). Interaction of childhood maltreatment with the corticotropin-releasing hormone receptor gene: effects on hypothalamic-pituitary-adrenal axis reactivity. Biol. Psychiatry 66, 681-685.

Vamvakopoulos, N. C., and Chrousos, G. P. (1993). Evidence of direct estrogenic regulation of human corticotropinreleasing hormone gene expression. J. Clin. Invest. 92, 1896-1902.

Weiser, M. J., Goel, N., Sandau, U. S., Bale, T. L., and Handa, R.J.(2008).Androgen hormone receptor 2 (CRHR2) mRNA expression and receptor binding in the rat brain. Exp. Neurol. [Epub ahead of print].

Weiss, E. L., Longhurst, J. G., and Mazure, C. M. (1999). Childhood sexual abuse as a risk factor for depression in women: psychosocial and neurobio816-828.

Conflict of Interest Statement: The authors disclose the following commercial or financial relationships that could be construed as a potential conflict of interest.

Dr. Heim has received research support from National Institutes of Health, National Alliance for Research on Schizophrenia and Depression, Anxiety Disorders Association of America, Center for Behavioral Neuroscience, Novartis, Eli Lilly, Centers for Disease Control, and Deutsche Forschungsgemeinschaft. She has served on speakers' bureaus and/or received honoraria Forest Laboratories, Wyeth, and Bristol-Myers-Squibb.

Dr. Bradley receives grant support or has received awards from AFSP and the American Psychoanalytic Association Psychoanalytic Research Fund.

Tanja Mletzko and Tod Deveau have no conflicts of interest, financial or otherwise, to declare. regulation of corticotropin-releasing logical correlates. Am. J. Psychiatry 156, 
Dr. Musselman had received grants from Forest Laboratories, GlaxoSmithKline, Janssen Pharmaceutica, Dana Foundation, NIMH, National Heart, Lung, and Blood Institute (NHLBI), and Schering Plough. Dr. Musselman served on the Speaker's Bureau of Forest Laboratories.

In the past, Dr. Nemeroff consulted to, served on the Scientific Advisory Board and/or Board of Directors, has been a grant recipient, and/or owned equity in one or more of the following: Abbott Laboratories, Acadia Pharmaceuticals, American Foundation for Suicide Prevention( AFSP), American Psychiatric Institute for Research and Educations(APIRE), AstraZeneca, BMC-JR LLC, Bristol-Myers-Squibb, CeNeRx, Corcept, Cypress Biosciences,
Cyberonics, Eli Lilly, Forest Laboratories, George West Mental Health Foundation, GlaxoSmithKline, i3 DLN, Janssen Pharmaceutica, Lundbeck, National Alliance for Research on Schizophrenia and Depression( NARSAD), Neuronetics, NIMH,NFMH, NovaDel Pharma, Otsuka, Pfizer Pharmaceuticals, Quintiles, Reevax, UCB Pharma, Wyeth-Ayerst. Currently, Dr. Nemeroff serves on the Scientific Advisory Board (SAB) for the American Foundation for Suicide Prevention (AFSP); AstraZeneca; NARSAD, PharmaNeuroboost and CeNeRx. He serves on the Board of Directors of American Foundation for Suicide Prevention (AFSP); George West Mental Health Foundation; NovaDel Pharma, Mt. Cook Pharma, Inc. He owns equity or is stock holder in Corcept; Revaax; NovaDel
Pharma; CeNeRx, PharmaNeuroboost, Mt. Cook Pharma. He is inventor on the following patents: Method and devices for transdermal delivery of lithium (US $6,375,990$ B1) and Method to estimate serotonin and norepinephrine transporter occupancy after drug treatment using patient or animal serum (provisional filing April, 2001).

Dr. Ressler has received awards and/or funding support related to other studies from Lundbeck, Burroughs Wellcome Foundation, Pfizer, NARSAD, NIMH, NIDA, and has a consulting agreement with Tikvah Therapeutics for NMDAbased therapeutics.

Dr. Binder receives grant support or has received research awards from NARSAD, the Doris Duke foundation, Pfizer, Pharma Neuroboost and NIMH.
Received: 09 July 2009; paper pending published: 16 August 2009; accepted: 09 October 2009; published online: 06 November 2009. Citation: Heim C, Bradley B, Mletzko TC, Deveau TC, Musselman DL, Nemeroff $C B$, Ressler KJ and Binder EB (2009) Effect of childhood trauma on adult depression and neuroendocrine function: sex-specific moderation by CRH receptor 1 gene. Front. Behav. Neurosci. 3:41. doi: 10.3389/neuro.08.041.2009

Copyright $@ 2009$ Heim, Bradley, Mletzko, Deveau, Musselman, Nemeroff, Ressler and Binder. This is an open-access article subject to an exclusive license agreement between the authors and the Frontiers Research Foundation, which permits unrestricted use, distribution, and reproduction in any medium, provided the original authors and source are credited. 